\title{
Exposure in vivo versus pain-contingent physical therapy in complex regional pain syndrome type I
}

Citation for published version (APA):

den Hollander, M., Heijnders, N., de Jong, J. R., Vlaeyen, J. W. S., Smeets, R. J. E. M., \& Goossens, M. E. J. B. (2018). Exposure in vivo versus pain-contingent physical therapy in complex regional pain syndrome type I: A cost-effectiveness analysis. International Journal of Technology Assessment in Health Care, 34(4), 400-409. https://doi.org/10.1017/S0266462318000429

Document status and date:

Published: 01/01/2018

DOI:

10.1017/S0266462318000429

Document Version:

Publisher's PDF, also known as Version of record

Document license:

Taverne

Please check the document version of this publication:

- A submitted manuscript is the version of the article upon submission and before peer-review. There can be important differences between the submitted version and the official published version of record.

People interested in the research are advised to contact the author for the final version of the publication, or visit the DOI to the publisher's website.

- The final author version and the galley proof are versions of the publication after peer review.

- The final published version features the final layout of the paper including the volume, issue and page numbers.

Link to publication

\footnotetext{
General rights rights.

- You may freely distribute the URL identifying the publication in the public portal. please follow below link for the End User Agreement:

www.umlib.nl/taverne-license

Take down policy

If you believe that this document breaches copyright please contact us at:

repository@maastrichtuniversity.nl

providing details and we will investigate your claim.
}

Copyright and moral rights for the publications made accessible in the public portal are retained by the authors and/or other copyright owners and it is a condition of accessing publications that users recognise and abide by the legal requirements associated with these

- Users may download and print one copy of any publication from the public portal for the purpose of private study or research.

- You may not further distribute the material or use it for any profit-making activity or commercial gain

If the publication is distributed under the terms of Article $25 \mathrm{fa}$ of the Dutch Copyright Act, indicated by the "Taverne" license above, 


\section{EXPOSURE IN VIVO VERSUS PAIN- CONTINGENT PHYSICAL THERAPY IN COMPLEX REGIONAL PAIN SYNDROME TYPE I: A COST-EFFECTIVENESS ANALYSIS}

Marlies den Hollander

Department of Clinical Psychological Science, Maastricht University Department of Rehabilitation, Maastricht University Medical Center Adelante Centre of Expertise in Rehabilitation marlies.denhollander@maastrichtuniversity.nl

Noortje Heijnders

Adelante Centre of Expertise in Rehabilitation

Jeroen R. de Jong

Department of Rehabilitation, Maastricht University Medical Center Adelante Centre of Expertise in Rehabilitation

CAPHRI - School for Public Health and Primary Care, Department of Rehabilitation Medicine, Maastricht University
Johan W.S. Vlaeyen

Department of Clinical Psychological Science, Maastricht University

Research Group Health Psychology, KU Leuven -University of Leuven

Rob J.E.M. Smeets

CAPHRI - School for Public Health and Primary Care, Department of Rehabilitation Medicine, Maastricht University

Libra Rehabilitation and Audiology, Eindhoven/Weert

Mariëlle E.J.B. Goossens

Department of Clinical Psychological Science, Maastricht University

CAPHRI - School for Public Health and Primary Care, Department of Rehabilitation Medicine, Maastricht University

Objectives: The aim of this study was to evaluate the cost-effectiveness of exposure in vivo (EXP, a cognitive-behavioral treatment targeting pain-related fear) in Complex Regional Pain Syndrome Type I (CRPS-I), as compared to pain-contingent physical therapy (PPT).

Methods: Data from a randomized controlled trial were used to compare the cost-effectiveness of EXP versus PPT from a societal perspective. Intervention costs, other healthcare costs, costs to patient and family, and productivity losses were included. The main outcomes were changes in the SF-36 physical component scale and quality-adjusted life-years. Changes were followed until 6 months after treatment. Uncertainty was estimated using nonparametric bootstrap analysis, cost-effectiveness acceptability curves and costeffectiveness planes. Sensitivity analyses were performed to check robustness of findings.

Results: Forty-six patients were randomized and thirty-eight completed the study. Over 6 months, EXP resulted in greater improvement in physical health-related quality of life and quality-adjusted life-years than PPT. Despite higher initial treatment costs, EXP showed a tendency to reduce all costs compared with PPT; healthcare costs were significantly reduced. Furthermore, the cost-effectiveness planes were in favor of EXP. Sensitivity analyses, for different program costs and complete cases only, confirmed robustness of these findings.

Conclusions: EXP, a cognitive-behavioral treatment, seems more costeffective than PPT in CRPS patients with pain-related fear. The initial higher costs for EXP are offset by a long-term reduction of costs for healthcare use, and a tendency to lower work absenteeism and reduced societal costs. Due to low sample sizes, replication of findings is required to confirm results.

Keywords: (RPS-I, Cost-effectiveness analysis, Pain-related fear, Exposure in vivo, Rehabilitation

We are grateful for the support of all individuals with CRPS-I who participated in the study, specialists and therapists, our research assistants, referrers to the trial and staff. Ethical standards: The authors assert that all procedures contributing to this work comply with the ethical standards of the relevant national and institutional committees on human experimentation and with the Helsinki Declaration of 1975, as revised in 2008. Financial support: This work was supported by the Profileringsfonds azM (PF 261); governmental funding for Maastricht University, Faculty of Psychology; the Research Foundation Flanders, Belgium, (J.V., Odysseus Grant G090208N "The Psychology of Pain and Disability Research Program"); and the Flemish Government, Belgium (J.V., "Asthenes" long-term structural funding - Methusalem grant METH/15/011). Funders had no role in study design, data collection and analysis, decision to publish, or preparation of the manuscript. The study sponsor had no role in the study design, collection, analysis, and interpretation of data, the writing of the report, or in the decision to submit for publication. The corresponding author had full access to all data and final responsibility for the decision to submit for publication.
Complex regional pain syndrome type I (CRPS-I) is a chronic pain condition affecting one of the limbs, without peripheral nerve injury, and usually after an injury or trauma. In addition to persistent pain, disproportionate in severity to any underlying observable pathology, other signs and symptoms may occur. These include allodynia, profound sweating, abnormal vascular reactivity, and functional impairment (1). Despite the many available medical treatments, there is a critical lack of high quality evidence for the effectiveness of most treatments (2). Especially in chronic CRPS-I, there is no treatment that guarantees cure or complete pain relief.

As a consequence, individuals continue to receive intensive medical care, and they continue to suffer from extensive 
limitations in daily life activities and social participation $(3 ; 4)$. This ongoing healthcare usage is expensive for patients and society. Productivity losses due to CRPS-I $(3 ; 4)$ seem to have an even higher economic impact. Several studies reporting on work-related disability rates (5-7) showed percentages of individuals completely unable to work varying between four (5) (United States) and thirty-one (6) (the Netherlands). A large percentage of individuals with CRPS-I are unable to perform household activities as well, resulting in dependence on domestic care (7).

Pain-related fear (PRF) has been identified as a predictor of poor outcome in CRPS-I (8). PRF has been consistently associated with more severe disability (9), and predicts the progression of disability over time (10). Exposure in vivo (EXP) is a cognitive-behavioral treatment that specifically targets PRF. Studies have shown that EXP not only reduces PRF, but also improves daily life functioning (11). A recent randomized controlled trial (RCT) $(n=46)$ showed that EXP is more successful in reducing disability than the pain-contingent physical therapy (PPT) recommended by the previous Dutch Guidelines (12) for CRPS-I patients with elevated levels of PRF. Six months after EXP, 94 percent of participants reported a reliable improvement in disability; in PPT this was only 18 percent. EXP was also superior in reducing pain intensity, perceived harmfulness of activities, and pain catastrophizing, and in increasing quality of life (QoL) (12). A remaining question is whether the application of EXP is also good value for money from a societal perspective. A positive economic evaluation would further support EXP. Therefore, we used data from the aforementioned RCT to examine the cost-effectiveness (CEA) and cost-utility (CUA) of EXP compared with PPT, in individuals with chronic CRPS-I and elevated levels of PRF.

\section{METHODS}

\section{Study Design and Participants}

This economic evaluation uses data derived from a previously published RCT (12) comparing the effects of EXP with PPT. The evaluation consists of a cost-effectiveness analysis and a cost-utility analysis, performed from a societal perspective with a time horizon of 6 months after completion of treatment. Individuals were included if they were between 18 and 65 years old. A physiatrist enrolled participants, after having confirmed the diagnosis of CRPS-I using the IASP Orlando criteria. Individuals had to report disability despite former treatment, and at least moderate levels of perceived harmfulness of activities, established with the Photograph Series of Daily Life Activities (PHODA score $\geq 34$ ).

Exclusion criteria were: insufficient comprehension of the Dutch language, pregnancy, CRPS-I in both legs/both arms, generalized pain, dystonia, involvement in a litigation procedure regarding CRPS-I, and severe psychopathology. The
Medical Ethics Committee of the University Hospital Maastricht (METC-azM reg.nr.20067) approved the study protocol, and the trial was registered (NCT00625976). All participants provided written informed consent. A predetermined and computer-generated randomization schedule, only accessible by one researcher, was used to allocate participants to EXP or PPT. Participants were informed about the allocated treatment during the first treatment session (12).

\section{Interventions and Setting}

We compared two active treatment approaches. EXP, a cognitive-behavioral intervention, widely applied to a variety of anxiety disorders (13) was compared with PPT, the recommended physical therapy treatment according to the Dutch Guidelines for CRPS-I at the onset of the trial. Therapists were specifically trained to deliver treatment according to the protocols for either EXP or PPT. To prevent contamination, therapists who delivered EXP did not deliver PPT, and vice versa. Extensive treatment manuals were provided for each condition. All treatments were delivered in a tertiary care rehabilitation center. A physiatrist was responsible for medical examination and deciding whether to include patients for participation in rehabilitation, and provided supervision to the team (EXP) or physical therapist (PPT) that delivered the intervention.

\section{Intervention: Exposure In Vivo (EXP)}

EXP specifically aims at improving functional ability through the violation of negative expectations about the relation between movement and increase in signs and symptoms. In various chronic pain syndromes, for example, low-back pain (14), whiplash associated disorder (15), and complaints of arm, neck, and shoulder (CANS) (16), EXP has been shown to be effective in reducing PRF and disability. EXP is characterized by systematic and repeated exposure to feared movements, activities and/or sensations to test and challenge the participants' catastrophic interpretations regarding these stimuli. EXP is described in detail elsewhere (11). A team consisting of a psychologist/behavioral therapist (PSY/BT) and an occupational or physical therapist (OT/PT), delivered EXP.

During each 1-hour session of EXP, both the PSY/BT and OT/PT assigned to the patient are present, working simultaneously and interdisciplinarily according to the protocol of EXP. In the first session, a cognitive-behavioral analysis of the pain problem is made. In the second session, the PHODA is used to reflect on specific catastrophic cognitions a patient might have about the activities shown in the pictures of the PHODA. In the third session, the therapists, while using the fear avoidance model, construct a personalized fear avoidance model with the patient. This model shows how the patient's cognitions/fears fuel the vicious circle of pain and disability. In the remaining sessions, the patient is exposed to movements, activities, and sensations of which the patient has fearful/ 
catastrophic cognitions to test and correct these expectations. Next, the patient is stimulated to perform these activities in his or her daily life, for example, at home, work, and leisure. All therapists were specifically trained to deliver EXP.

\section{Comparator: Pain-Contingent Physical Therapy (PPT)}

In CRPS-I of less than 1 year, PPT was proven to be more effective in reducing pain, signs, and symptoms than an active control treatment with occupational therapy and a social-therapy control treatment (17). PPT is a pain-contingent treatment protocol, aiming to increase control over pain and optimize coping with CRPS-I. After categorization of the individual's current level of control over pain (low, moderate or high), treatment may focus on extinguishing the source of ongoing pain by rest (locally), connective tissue massage, transcutaneous electric nerve stimulation (TENS), and painreducing exercises (directed to stimulate kinetic receptors type I and II). PPT also aims to improve skills by practicing compensatory activities with instructions regarding ergonomic positioning of the body. PPT was delivered by a physical therapist. Therapists received specific training to deliver PPT.

\section{Data Collection}

Demographics (age, gender, education level, employment status, and pain duration) were recorded before the start of treatment. Outcome measures (SF-36-PCS, PHODA) were collected electronically during four assessments at Maastricht University; twice at baseline (two identical premeasurements within 2 weeks before the start of treatment, a mean score being calculated), directly after completion of treatment (posttreatment) and 6 months after treatment (follow-up). Cost measures were completed from 4 weeks before baseline until 6 months after completion of treatment.

\section{Cost Measures}

To evaluate the economic consequences of treatment from a societal perspective, we assessed the intervention costs, other healthcare costs, patient and family costs, and productivity losses.

Intervention costs were based on the 17 hours of treatment that each patient received according to the protocol. Both treatments were delivered in a rehabilitation setting (with extensive facilities, e.g., therapeutic pool, different gyms, sports hall) under medical supervision of a physiatrist. In the Netherlands standardized costs prices are used for rehabilitation treatment. To calculate treatment costs for EXP and PPT, we used these standardized cost-prices as prescribed in the Dutch manual for cost-analysis in healthcare research (18). For EXP, we added the costs of a psychologist to this standardized rehabilitation price (SRP), to reflect the presence of two therapists working with one patient at the same time.

Other healthcare costs concerned all CRPS-I-related healthcare usage, including visits to the general practitioner, medical specialists, hospitalization, professional care, therapeutic consultations, prescribed and/or over-the-counter medication, and alternative healthcare. Patient and family costs included outof-pocket costs, such as costs for home care, informal care, and additional expenses. Productivity losses were calculated based on days absent from paid and unpaid work due to CRPS-I.

Participants used a cost diary to register healthcare usage (except the intervention), help received from family and/or friends and productivity losses (19). Participants completed this cost diary weekly (at home), starting from 4 weeks pretreatment until 6 months after completion of treatment. Every 4 weeks, the diaries had to be returned in a sealed coded return envelope. Research assistants contacted participants if they did not return their diaries on time.

Total costs were estimated using a bottom-up approach, where information on each element of service used was multiplied by an appropriate standardized unit cost and added up to arrive at an overall total cost (20). For the cost valuation, we used standardized cost-prices from the Dutch manual for cost-analysis in healthcare research (18). Costs for prescribed medication were calculated by multiplying the number of boxes that participants reported in their cost diary, with the price per box provided by the Dutch website www.medicijn kosten.nl. For over-the-counter medication, participants did not provide the number of boxes they used, so we calculated the costs for a standard unit of use as handled by the drugstore and pharmacy. Where medication prices for prescribed- or overthe-counter medication varied, the lowest cost price was used. Additional prescription charges for prescribed medication were also taken into account.

Travel expenses were calculated based on the standardized mean distance to a specific healthcare provider in the Netherlands, multiplied by standard cost-prices from the Dutch manual for cost-analysis in healthcare research (18). Cost-prices for alternative healthcare providers were derived from the ALASCA trial (21), as well as CRPS-I-related activities and sports. We calculated prices of informal care using standardized cost prices based on general hourly wages (18). Additional expenses were determined using the costs participants recorded in their cost diaries; these expenses included orthopedic shoes, braces, wheelchair, voice-recognition software, and car adjustments.

To calculate productivity losses, we used a Human Capital Approach (22), multiplying a standardized cost-price for mean productivity cost per hour per paid employee by the hours absent from work (18). All costs are presented in euros, using the 2015 price level. If necessary, prices were indexed to 2015 using rates from Statistics Netherlands.

\section{Outcome Measures}

For the cost-effectiveness of this economic evaluation, the physical component scale of the SF-36 was used as primary outcome 
(this differs from the primary outcome used in the RCT; because in the RCT, upper and lower extremity disability were measured separately with their own specific questionnaire, it was not possible to use these questionnaires to calculate overall economic consequences). The SF-36 is a generic QoL questionnaire, reflecting eight domains of perceived health. Higher scores reflect a better health-related QoL. A Dutch translation of the SF-36 was found to be reliable and valid (23). Four domains (physical functioning, physical role limitation, bodily pain, general health perceptions) yield a physical health component scale (SF-36-PCS). The domains vitality, social functioning, emotional role limitations, and general mental health form a mental health component scale (SF-36-MCS).

These summary measures are supported by factor analyses (24). For the cost-utility analysis, we used quality-adjusted lifeyears (QALYs) as primary outcome. First, utilities were derived using an algorithm of the SF-6D, which transforms the healthrelated QoL preferences (SF-36) into generic utilities scores. Utilities are values assigned to a particular qualitative health status and are expressed on a scale from 0-1 (25). Higher scores reflect a better health. QALYs were calculated using the derived utilities at the three measurement points multiplied by the amount of time spent in that health state ("area under the curve" method) (26).

\section{Statistical Analysis}

An intention-to-treat principle was used. When both pretreatment assessments (SF-36 and PHODA) revealed no significant differences at baseline, the mean of both measurements was calculated. Participants who completed at least three cost diaries, as well as pre- and posttreatment measurement (for the main outcomes), were included in the economic analysis. In case of missing outcomes or cost diaries, we chose imputation of the individual mean. Because differences between participants were larger than differences within participants, we anticipated that this method would yield the most reliable estimate (27). When follow-up measurement took place longer than 10 months after baseline measurement, actual data were standardized to capture the costs over a 10-month period of time.

When a main outcome (SF-36-PCS) was missing at followup measurement ( 6 months posttreatment), we used the lastobservation-carried-forward method (e.g., posttreatment score was used as 6 months after treatment score). We checked whether dropout was selective by analyzing if any significant differences occurred at baseline between dropouts and completers for age, gender, educational level, duration of complaints, or health-related QoL (SF-36-PCS/MCS). Group differences were calculated using chi-squared tests (categorical variables: gender, education) and $t$-tests (continuous variables: age, duration of complaints, health-related QoL).

Outcomes on questionnaires were compared using $t$-tests in IBM SPSS Statistics version 22. Costs were compared using bootstraps (1,000 replications) with Microsoft Excel 2010 with mean difference and 95 percent confidence interval. Additionally, bootstrapping with Microsoft Excel 2010 was used to explore sample uncertainty $(5,000$ replications $)$ around the incremental cost-effectiveness ratio (ICER). Nonparametric bootstrapping is a method based on random sampling with replacement based on the individual data of participants. The ICER is defined by the difference in costs between EXP and PPT, divided by the difference in their effect. Cost-effectiveness analyses were performed by relating the mean total costs to the health-related QoL physical component (SF-36-PCS) score at 6 months after treatment.

A cost-utility analysis was performed by relating the mean total costs to the mean health-utility (QALY) scores of both groups. The bootstrapped ICERs were subsequently plotted in cost-effectiveness planes consisting of four quadrants. The southeast quadrant represents a more effective intervention that is less costly than usual care, the so-called dominant quadrant. The northwest quadrant represents inferiority: a costlier intervention that is also less effective. In the northeast quadrant, the ICER indicates the additional amount that must be paid to gain one unit of health, and ICERs below the ceiling ratio are considered to be cost-effective. In contrast, the ICER in the southeast quadrant indicates the savings gained for a one-unit loss of health. Subsequently, treatments with high ICERs are preferable to those with low ICERs (more money saved for the same loss of health) and only those treatments with ICERs above the ceiling ratio should be adopted.

Additionally, cost-effectiveness acceptability curves (CEACs) were constructed to show the probability that the intervention was cost-effective, given different ceiling ratios. Only treatments resulting in costs between $€ 16,000$ (for the lowest disease burden) and $€ 80,000$ (for the highest burden) per QALY are considered for reimbursement according recommendations of the Dutch Council for Public Health and Healthcare.

\section{Sensitivity Analyses}

Two sets of sensitivity analyses were performed to measure the robustness of the economic evaluation. Because several assumptions were made for the calculation of the costs, uncertainty could have been introduced in the analyses. The sensitivity analyses explore the impact of the assumptions on the results from changing the value of one parameter while keeping the values of all other parameters unchanged (28). To improve generalizability to other healthcare settings, we first performed sensitivity analyses with different treatment costs, to reflect that EXP and PPT can be delivered in other healthcare settings against different treatment costs.

We performed one sensitivity analysis with the costs for rehabilitation (for EXP), versus a physical therapist in primary care (for PPT), because PPT can be delivered as a primary care intervention. In the second sensitivity analysis, 
we used the costs for rehabilitation plus an extra psychologist (EXP) versus a physical therapist in primary care for PPT, to capture the maximum contrast in treatment costs between PPT (when delivered as primary care) and EXP (delivered as an interdisciplinary treatment simultaneously delivered by a psychologist and a PT/OT). In the second set of sensitivity analyses, we used data from the complete cases only. A case was considered complete when a participant had completed a minimum of eight cost diaries.

\section{RESULTS}

\section{Participants and Comparability}

Forty-six individuals were included: twenty-three participants were randomized to each condition. The CONSORT flow chart, including number of dropouts per phase with reasons is presented elsewhere (12). Three participants dropped out between allocation and start of treatment (one EXP, two PPT), and five during treatment (three EXP, two PPT). These participants did not complete posttreatment measurements nor did they complete enough diaries to be included in the

Table 1. Mean (SD) in Demographic Characteristics, Duration of Complaints, and Disability at Baseline per Treatment Condition

\begin{tabular}{|c|c|c|}
\hline \multirow[b]{2}{*}{ Variables } & \multicolumn{2}{|c|}{ Mean (SD) } \\
\hline & $\operatorname{EXP}(n=19)$ & PPT $(n=19)$ \\
\hline Age, mean (SD) & $46.26(12.08)$ & $42.63(10.83)$ \\
\hline Gender $\%$ male $(n)$ & 21.1 (4 of 19) & $10.5(2$ of 19$)$ \\
\hline \multicolumn{3}{|l|}{ Education } \\
\hline$\%$ Low $(n)$ & $52.6(10)$ & $63.2(12)$ \\
\hline$\%$ Middle $(n)$ & $31.6(6)$ & $26.3(5)$ \\
\hline$\%$ High $(n)$ & $15.8(3)$ & $10.5(2)$ \\
\hline \multicolumn{3}{|l|}{ Work status } \\
\hline \% Working full contract hours ( $n$ ) & $5.3(1)$ & $5.3(1)$ \\
\hline \% Partly on sick leave $(n)$ & $31.6(6)$ & $36.8(7)$ \\
\hline \% Completely on sick leave $(n)$ & $5.3(1)$ & $36.8(7)$ \\
\hline \% Disability pension $(n)$ & $47.4(9)$ & $15.8(3)$ \\
\hline$\%$ Other $(n)$ & $10.5(2)$ & $5.3(1)$ \\
\hline $\begin{array}{l}\text { Mean duration of complaints in years (SD) } \\
\text { Health-related quality of life (SF-36) (0-100) }\end{array}$ & $5.93(6.02)$ & $3.74(4.83)$ \\
\hline Physical component subscale (SF-36) & $40.60(13.39)$ & $41.86(16.30)$ \\
\hline Mental component subscale (SF-36) & $57.08(14.03)$ & $63.92(19.44)$ \\
\hline PHODA (0-100) & $64.51(16.49)$ & 65.67 (17.02) \\
\hline Utility $(0-1)$ & $0.53(0.05)$ & $0.51(0.06)$ \\
\hline
\end{tabular}

$\mathrm{Cl}$, confidence interval; EXP, exposure in vivo; PHODA, Photographic Series of Daily Activities (a lower score indicates less perceived harmfulness of activities); PPT, paincontingent physical therapy; SD, standard deviation; SF36, Short Form 36 (higher scores indicate a better health-related QoL). economic analysis. Baseline characteristics (age, gender, level of education, pain duration, and health-related QoL) of those who dropped out did not significantly differ from participants who completed participation in the study (all $p>.10$, data not presented).

Thirty-eight participants were included in the economic analysis (nineteen EXP, nineteen PPT). The mean number of diaries completed did not significantly differ between conditions; EXP 10.11 (SD 3.38) and PPT 10.00 (SD 2.75), mean difference 0.105 (confidence interval [CI], -1.92 to 2.13 ). We imputed data (last-observation-carried-forward method) for participants $(n=3)$ whose main outcomes were missing at 6 months posttreatment measurement.

The mean age of the thirty-eight participants was 44.4 years $(\mathrm{SD}=11.47) ; 84$ percent were women. At the start of the study, two participants worked their regular contract hours, twentyone participants were on sick leave (eight completely, thirteen partly), twelve received disability pension, one studied, and two were unemployed (not due to CRPS-I). The mean duration of CRPS-I was 5.1 years $(\mathrm{SD}=5.45)$. Table 1 presents baseline characteristics and costs for EXP and PPT. Chi-squared tests revealed no significant differences between EXP and PPT regarding demographic variables. Independent samples $t$-tests showed no significant baseline differences between conditions for duration of complaints, PRF, and health-related QoL (all $p>.10$, results not reported). Table 2 presents baseline costs for EXP and PPT. Bootstrapping of the baseline costs revealed no significant differences between conditions before the start of treatment.

\section{Costs and Effects}

After completion of treatment, EXP participants use significantly less prescribed (between group difference, -11.30 ; CI, -21.02 to -1.57 ) and over-the-counter medication (between group difference, -1.93 ; CI, -3.74 to -0.13 ) than PPT participants. Furthermore, EXP participants had, although not statistically significant, fewer visits to medical specialists, and regular visits (physical/occupational therapists and

Table 2. Mean (SD) Cost Components (in EUR) per Treatment Condition over a 4Week Baseline Period

\begin{tabular}{lcc}
\hline & \multicolumn{2}{c}{ Mean (SD) } \\
\cline { 2 - 3 } Variables & $\operatorname{EXP}(n=19)$ & PPT $(n=19)$ \\
\hline Healthcare costs & $271.79(180.71)$ & $553.90(255.68)$ \\
Patient and family costs & $205.58(74.76)$ & $225.38(79.47)$ \\
Productivity loss & $2687.32(616.73)$ & $3449.10(496.57)$ \\
Total costs & $3178.09(758.69)$ & $4220.89(635.05)$ \\
\hline
\end{tabular}

EXP, exposure in vivo; PPT, pain-contingent physical therapy; SD, standard deviation. 
psychologists), as well as to alternative healthcare providers, compared with participants who received PPT. The number of visits to the general practitioner is in favor of PPT. No participants were hospitalized due to CRPS-I after EXP, whereas one was after PPT. After EXP, participants needed fewer hours of home care and domestic care, and significantly fewer hours of help from family (between group difference, -61.22 ; CI, -116.31 to -6.12 ). Participants receiving EXP were less absent from paid work, but more from usual activities and unpaid work than participants who received PPT, although these differences were not statistically significant.

Supplementary Table 1 shows the mean resource usage per participant for EXP and PPT. Table 3 provides an overview of outcomes and the overall societal costs six months after completion of treatment for both conditions. Because pretreatment measures (SF-36-PCS and MCS) revealed no significant differences at baseline, a mean score was calculated. At the follow-up measurement 6 months after treatment, participants receiving EXP showed significantly higher scores on the physical component scale of the SF-36 and a higher QALY compared with participants receiving PPT (Table 3). The utility score was in favor of EXP, but the difference with PPT was not significant. Participants who received EXP had significantly lower healthcare costs than participants receiving PPT (Table 3). Costs for productivity loss, as well as patient and family costs, were in favor of EXP, although the differences were not significant.

Overall, the total societal costs for participants receiving EXP were lower than for participants receiving PPT (€28,446 vs. $€ 43,177)$, but bootstrap replications revealed no significant differences between the groups. Productivity loss was the main source of costs in both groups and accounted for approximately 77 percent of the total costs (EXP 80 percent, PPT 76 percent).

\section{Cost-Utility and Cost-Effectiveness Planes and CEACs}

EXP resulted in a larger health benefit (26.33 points improvement on SF-36 PCS from pre- to posttreatment) than PPT (4.05 points), and, although treatment costs for EXP were higher than for PPT ( $€ 3399$ vs. $€ 2627$, difference $€ 772$ ), the total societal costs six months after treatment clearly favored EXP. Figure 1 shows the cost-effectiveness plane (5,000 bootstrap replications) and CEAC for the SF-36 PCS. EXP clearly resulted in a better outcome than PPT: 99.7 percent of the bootstrapped cost-effect pairs are located in the eastern part of the plane, implicating higher effects. The majority of these pairs ( 94.5 percent) is located in the southeast quadrant, indicating not only higher effects, but also lower costs. The north-east quadrant contains the remaining 5.2 percent of the replications, indicating higher effects but higher costs.

The CEAC, based on 1-point improvement on the SF-36 PCS, shows that the probability of the intervention being cost-effective is 100 percent. To gain 1 additional unit on the SF-36 PCS, it does not matter if the highest $(€ 80,000)$ or lowest $(€ 16,000)$ threshold value is chosen: EXP is more cost-effective than PPT. Supplementary Figure 1 shows the cost-effectiveness plane (5,000 bootstrap replications) and the CEAC for the QALY. EXP resulted in a greater health benefit than PPT: 99.7 percent of the bootstrapped ICERs are located in the eastern part of the plane, implicating higher effects. The majority of the ICERs (95.1 percent) lies in the southeast quadrant, indicating higher effects with lower costs. The northeast quadrant contains the remaining 4.6 percent of the replications, indicating higher effects but higher costs. The probability of the intervention being cost-effective ranges between 95 percent and 98 percent, depending on the threshold value of $€ 16,000$ and $€ 80,000$, respectively.

\section{Sensitivity Analyses}

Two sets of sensitivity analyses were performed: one with different treatment costs (recognizing that both EXP and PPT can be delivered in various healthcare settings leading to different cost-prices for treatment) and one with the complete cases only.

Sensitivity Analysis with Different Treatment Costs. In the first sensitivity analysis, the cost-utility analysis (QALY) was repeated using different intervention costs for PPT and EXP. In the first analysis, the reference cost for physical therapy in primary care in the Netherlands was used (PPT: $€ 1,133.22$ ), and compared with the reference price for rehabilitation treatment (EXP: €2,627.01). Again, the dominance of EXP (less disability at lower costs) was shown in 94.8 percent of the replications (southeast quadrant). In 5.1 percent of the replications, EXP is more effective, but against higher costs (northeast quadrant). The cost-effectiveness plane with CEAC is shown in Supplementary Figure 2.

In the second analysis, the reference cost for physical therapy in primary care was used again for PPT $(€ 1,133.22)$. For EXP, we now used the reference cost for rehabilitation with additional costs for a psychologist (€3,398.98). Still, 93.8 percent of the ICERs are located in the southeast quadrant, with 6 percent in the northeast quadrant. For the cost-effectiveness plane with CEAC, see Supplementary Figure 3.

Sensitivity Analysis with Complete Cases Only. In the complete case analysis, we considered participants who had completed at least eight diaries as complete cases (two EXP and four PPT participants were hence excluded) and repeated the cost-utility analysis (QALY). As in our primary analysis, we first used the SRP for PPT and SRP plus additional costs for a psychologist for EXP (Supplementary Figure 4). For the complete cases, 83.2 percent of the ICERs are located in the southeast quadrant, and 15.8 percent in the northeast quadrant. In 0.8 percent of the bootstraps, EXP was inferior. For the cost-effectiveness plane with CEAC, see Supplementary Figure 4. When the contrast between the costs for EXP versus PPT is maximized (costs 
den Hollander et al.

Table 3. Mean Scores at 6 Months Follow-up for Mean Outcome Variables and Total Costs (EUR) over Treatment- and Follow-up Period

\begin{tabular}{|c|c|c|c|c|}
\hline Variables & $\begin{array}{c}\text { Mean (SD) } \\
\operatorname{EXP}(n=19)\end{array}$ & PPT $(n=19)$ & $\begin{array}{c}\text { Mean difference }(95 \% \mathrm{Cl}) \\
\text { EXP vs PPT }\end{array}$ & $p$-Value \\
\hline $\begin{array}{l}\text { SF-36 PCS } \\
\text { Utility }(0-1)^{1} \\
\text { QALY' } \\
\text { Treatment costs } \\
\text { Healthcare costs } \\
\text { Patient and family costs } \\
\text { Productivity loss } \\
\text { Total }^{2} \text { costs }^{2}\end{array}$ & $\begin{array}{c}66.93(23.36) \\
0.70(0.13) \\
0.62(0.07) \\
3,398.98(0) \\
1,024.81(667.17) \\
1,514.18(705.53) \\
22,704.07(5,632.05) \\
28,446.04(6,322.10)\end{array}$ & $\begin{array}{cl}45.91 & (22.68) \\
0.62 & (0.15) \\
0.55 & (0.10) \\
2,627.01 & (0) \\
5,423.23 & (2,212.29) \\
2,667.79 & (845.44) \\
32,861.73 & (4,976.82) \\
43,177.29 & (5,946.42)\end{array}$ & $\begin{array}{c}21.02(5.87 \text { to } 36.17)^{\star} \\
0.08(-0.02 \text { to } 0.17) \\
0.07(0.02 \text { to } 0.13)^{\star} \\
771.97 \\
-4,398(-9,643 \text { to }-563)^{\star} \\
-1,154(-3,427 \text { to } 810) \\
-10,158(-25,192 \text { to } 4,461) \\
-14,731(-30,921 \text { to } 2,020)\end{array}$ & $\begin{array}{l}=.008 \\
=.101 \\
=.011\end{array}$ \\
\hline
\end{tabular}

1 Difference in mean (95\% confidence interval) derived with independent samples t-test.

2 Difference in mean (95\% confidence interval) derived with bootstrapping (1000 replications).

${ }^{*}$ significanlty indicates significant finding * $=$ sig.

Cl, confidence interval; EXP, exposure in vivo; PPT, pain-contingent physical therapy; QALY, quality-adjusted life-year (a higher score indicates a higher quality adjusted life year); SD, standard deviation; SF-36 PCS, Short Form-36 Physical Component Scale (a higher score indicates better physical health-related quality of life).

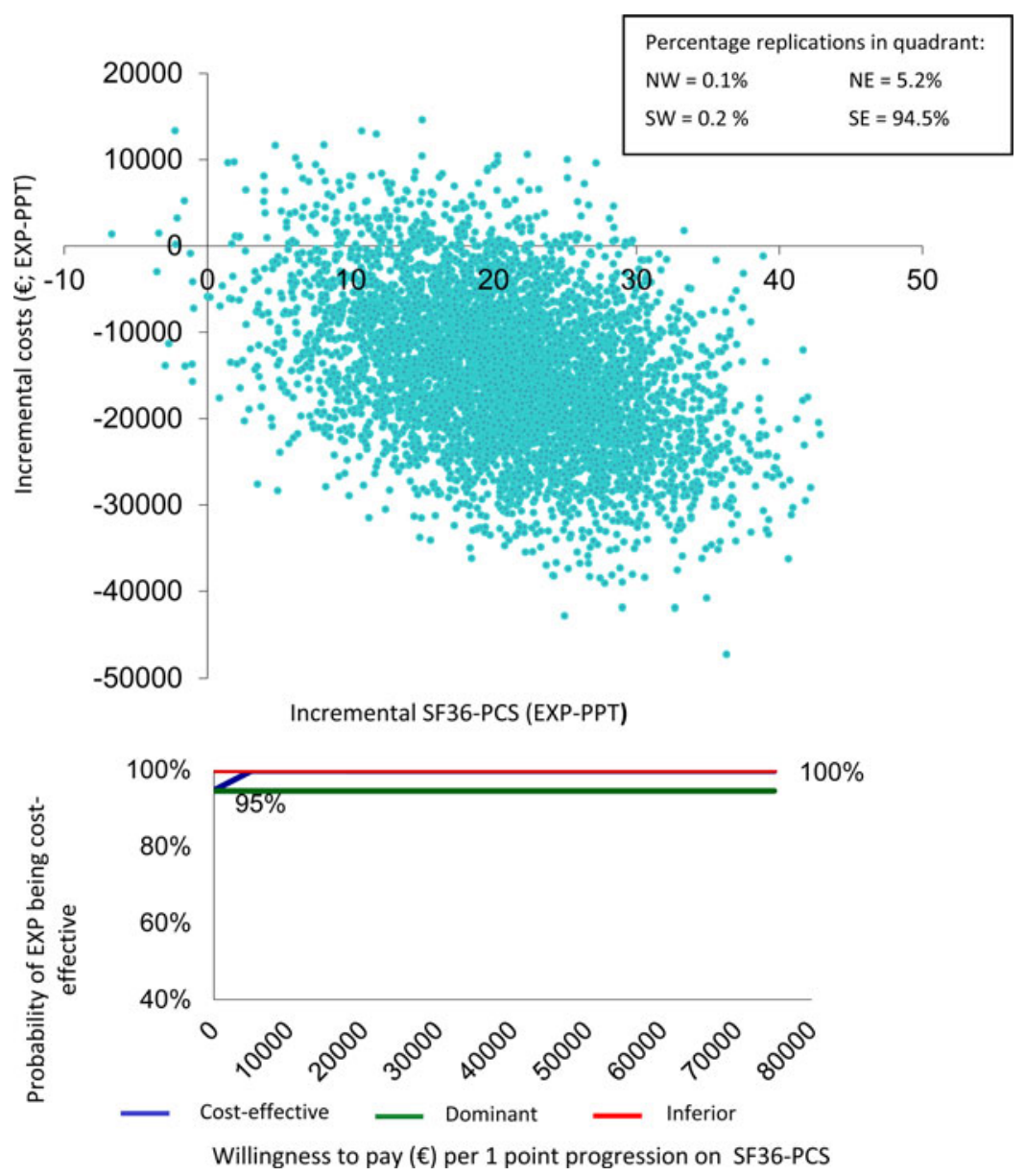

Figure 1. Cost-effectiveness plane and cost-effectiveness acceptability curve for SF-36-PCS for EXP (delivered as rehabilitation in tertiary care with additional costs for psychologist) versus PPT (delivered as rehabilitation in tertiary care). EXP, exposure in vivo; NE, northeast; NW, northwest; PPT, pain-contingent physical therapy; SE, southeast; SF-36-PCS, Short Form 36-Physical Component Scale; SW, southwest. 
for a primary care physical therapist versus SRP plus additional costs for a psychologist), 77.9 percent of the ICERs are located in the southeast quadrant, and 20.9 percent in the northeast quadrant. In 1 percent of the bootstraps, EXP was inferior. The cost-effectiveness plane with CEAC is shown in Supplementary Figure 5.

\section{DISCUSSION}

This economic analysis supports previous findings (12) that EXP seems superior to pain-contingent physiotherapy. EXP not only resulted in a greater improvement in physical healthrelated QoL and QALYs than PPT, EXP also seems to reduce societal costs six months after completion of treatment. This despite initially higher treatment costs due to the additional costs of a psychologist. After EXP, healthcare costs were significantly lower than after PPT. Participants used significantly less prescribed and over-the-counter medication after EXP, and needed significantly less informal care. In 95 percent of the bootstrap replications, EXP was more effective and had lower costs.

In CRPS-I, no other studies on the cost-effectiveness of rehabilitation are available. The cost-effectiveness of EXP was previously evaluated in a study comparing EXP with graded activity (GA) for individuals with low back pain (29). EXP was the preferred treatment regarding societal costs, but less convincing than in the current study, possibly because the contrast between EXP and GA, two biopsychosocial interventions, is less pronounced than between EXP and PPT, which has a biomedical focus.

We can explain the positive findings of lower costs for the individuals treated by EXP in two ways: the resumption of activities and the decrease in pain experience (12). First, EXP aims to specifically violate dysfunctional ideas about negative consequences of performing movements and activities. During EXP, individuals are exposed to the full range of activities they used to perform before the onset of CRPS-I, including work-related activities. EXP hereby facilitates and stimulates the resumption of personally relevant activities. When individuals resume self-care and household activities, they become less dependent upon professional care and need less help from their significant others.

The resumption of activities might also explain the positive findings for EXP on societal costs, because individuals are more able to return to work. Second, although EXP does not primarily focus on pain reduction, EXP did have pain-reducing effects (12;30;31). The current study extends these findings by showing that participants actually take less medication after EXP. An additional effect might be that, when individuals decrease their use of complex pain medication, they need fewer visits to their pain specialist. Actual pain reduction is probably not the only explanation for the decrease in medication use. By the violation of catastrophic assumptions and the reduction in fear, EXP aims to decrease the threat value of pain (32). As individuals catastrophize less about pain, signs, and symptoms after EXP, they may turn to their general practitioner when they do have CRPS-related questions, instead of turning to more expensive medical specialists, as is shown in our sample.

Participants receiving EXP had fewer hours of work absence than participants receiving PPT. At baseline, the number of participants working or being partly on sick leave was comparable over different conditions. In PPT, more individuals were on complete sick leave, whereas more EXP participants were already receiving a disability pension. In the Netherlands, only individuals on sick leave have the possibility of resuming work, because they are still under contract with their employer. Individuals on disability pension are paid by the government and are no longer employed: in consequence, they cannot just restart work even if willing and able to. Thus, EXP participants had less opportunity to improve on this outcome measure than PPT participants. It suggests that over time, the effect for EXP might even increase, as individuals with a disability pension regard themselves as being able to work again, or when the system establishes that they no longer need a disability pension and provides possibilities to resume work.

Kemler and Furnee (2002) showed that not only individuals with CRPS-I lose their jobs or decrease their working hours, but also that, for males with CRPS-I, spouses increased their working hours significantly (7). This again highlights the importance of performing a full economic analysis, including patient-related costs and indirect costs, instead of just comparing treatment costs that cannot make valid claims for financial benefits to society. Because societal costs due to work loss in participants were found to be 77 percent of the total economic costs, in line with cost-effectiveness studies in other chronic pain syndromes (29), treatments reducing work absenteeism are likely to have an enormous positive impact.

First, for individuals with CRPS-I, we showed that the value of EXP is that patients will reduce their use of medication and need less informal care. For healthcare insurance companies, EXP is shown to be good value for money, because the initial higher treatment costs clearly pay off in the long run through the reduction of medication and interventions, putting less burden on the total budget. For society in general, EXP enabled patients to stay at work and reduced the costs for work-disability. Policymakers should consider adopting EXP in (inter-)national guidelines for CRPS-I, because this specific protocol of EXP is shown to be effective and cost-effective.

Second, we performed sensitivity analyses to increase generalizability to other healthcare systems than the Dutch, by using different treatment costs reflecting different settings where treatment could be delivered. These sensitivity analyses yielded almost the same results, indicating that the effect is robust and not dependent on relatively small changes in initial treatment costs. Even with the maximal contrast in 
costs between treatments, primary care physical therapy against rehabilitation with additional costs for a psychologist, EXP remains the preferred treatment. To further reduce the costs of EXP, it would be worthwhile to investigate if it is possible to accomplish comparable effects in primary health care.

Several weaknesses have to be mentioned as well. First, we would have preferred to use disability as our primary outcome, as we did in the previously published RCT. However, because disability was measured for upper $(n=27)$ and lower limb $(n=11)$ CRPS-I separately, this would have resulted in very low power. Because studies are not powered for economic evaluations primarily, statistical power is always low, and we did not want to increase this problem even further. Instead, we chose the Physical Component Scale of the SF-36 as our primary measure of effect, reflecting a combination of disability and pain experience. This choice was justified by high correlations of the formerly used changes on the disability measures with changes in the SF-36 Physical Component Scale (-0.79 for both measures).

Second, participants did not always complete their cost diaries conscientiously, resulting in missing data. This forced us to impute data. We choose the most stringent procedure, imputation of the treatment mean (27). Because the percentage of missing data did not differ between conditions, this should have affected outcomes in both conditions equally. Additionally, a sensitivity analysis showed that even when only complete datasets were used, EXP still is more effective against lower cost than PPT (83 percent and 78 percent, respectively, depending on the treatment costs used).

Third, generalizability of our results to other countries might be limited, due to our use of a pain-contingent comparator (PPT), which might not be recommended by guidelines in other countries, as it was in the Netherlands. Future research is needed to draw conclusions about (cost-) effectiveness of EXP against other physiotherapeutic approaches, which may also incorporate principles that are more compatible with EXP and would thus decrease the contrast between treatments. Furthermore, costs are calculated based on the Dutch healthcare system. With our sensitivity analyses, we contrasted different healthcare settings where treatments could be delivered. Nevertheless, with the current contrast between EXP and PPT, we cannot draw conclusions about the cost-effectiveness of EXP against other biopsychosocial approaches in CRPS-I.

In conclusion, earlier findings on the effectiveness of EXP (12) are now corroborated with positive findings on its cost-effectiveness using a societal perspective, although the sample size of the trial was relatively small. The initial higher costs for EXP paid off in a significant long-term reduction of costs for healthcare consumption and a tendency to reduced costs for work absenteeism. Furthermore, participants needed less help from relatives after EXP. This would justify EXP being mentioned as a viable treatment in national and international guidelines for CRPS-I patients with PRF. Future research may replicate our findings in countries with different healthcare systems than the Netherlands, as well as with other comparator treatments.

\section{SUPPLEMENTARY MATERIAL}

The supplementary material for this article can be found at https://doi.org/10.1017/S0266462318000429

Supplementary Table 1: https://doi.org/10.1017/

S0266462318000429

Supplementary Figure 1: https://doi.org/10.1017/

S0266462318000429

Supplementary Figure 2: https://doi.org/10.1017/

S0266462318000429

Supplementary Figure 3: https://doi.org/10.1017/

S0266462318000429

Supplementary Figure 4: https://doi.org/10.1017/

S0266462318000429

Supplementary Figure 5: https://doi.org/10.1017/

S0266462318000429

\section{CONFLITS OF INTEREST}

The authors have nothing to disclose.

\section{REFERENCES}

1. Bruehl S, Harden RN, Galer BS, et al. Complex regional pain syndrome: Are there distinct subtypes and sequential stages of the syndrome? Pain. 2002;95:119-124.

2. O'Connell NE, Wand BM, McAuley J, et al. Interventions for treating pain and disability in adults with complex regional pain syndrome. Cochrane Database Sys Rev. 2013:CD009416.

3. Sharma A, Agarwal S, Broatch J, et al. A web-based cross-sectional epidemiological survey of complex regional pain syndrome. Reg Anesth Pain Med. 2009;34:110-115.

4. Geertzen J, Dijkstra P, Groothoff J, et al. Reflex sympathetic dystrophy of the upper extremity-a 5.5-year follow-up. Part I. Impairments and perceived disability. Acta Orthop Scand Suppl. 1998;279:12-18.

5. Sandroni P, Benrud-Larson LM, McClelland RL, et al. Complex regional pain syndrome type I: Incidence and prevalence in Olmsted county, a population-based study. Pain. 2003;103:199-207.

6. de Mos M, de Bruijn AGJ, Huygen FJPM, et al. The incidence of complex regional pain syndrome: A population-based study. Pain. 2007;129:12-20.

7. Kemler MA, Furnee CA. The impact of chronic pain on life in the household. J Pain Symptom Manage. 2002;23:433-441.

8. Bean DJ, Johnson MH, Heiss-Dunlop W, et al. Do psychological factors influence recovery from complex regional pain syndrome type 1? A prospective study. Pain. 2015;156:2310-2318.

9. Zale EL, Lange KL, Fields SA, et al. The relation between pain-related fear and disability: A meta-analysis. J Pain. 2013;14:1019-1030.

10. Zale EL, Ditre JW. Pain-Related fear, disability, and the fear-avoidance model of chronic pain. Curr Opin Psychol. 2015;5:24-30.

11. Vlaeyen JWS, Morley S, Linton SJ, et al. Pain-related fear: Exposurebased treatment for chronic pain. Washington, DC: IASP Press; 2012.

12. den Hollander M, Goossens MEJB, de Jong JR, et al. Expose or protect? A randomized controlled trial of exposure in vivo vs pain-contingent 
treatment as usual in patients with complex regional pain syndrome type 1. Pain. 2016;157:2318-2329.

13. Craske M, Treanor M, Conway $\mathrm{C}$, et al. Maximizing exposure therapy: An inhibitory learning approach. Behav Res Ther. 2014;58:10-23.

14. Leeuw M, Goossens ME, van Breukelen GJ, et al. Exposure in vivo versus operant graded activity in chronic low back pain patients: Results of a randomized controlled trial. Pain. 2008;138:192-207.

15. de Jong JR, Vangronsveld K, Peters ML, et al. Reduction of pain-related fear and disability in post-traumatic neck pain: A replicated single-case experimental study of exposure in vivo. J Pain. 2008;9:1123-1134.

16. de Jong JR, Vlaeyen JWS, van Eijsden M, et al. Reduction of pain-related fear and increased function and participation in work-related upper extremity pain (WRUEP): Effects of exposure in vivo. Pain. 2012;153:2109-2118.

17. Oerlemans HM, Oostendorp RA, de Boo T, et al. Pain and reduced mobility in complex regional pain syndrome I: Outcome of a prospective randomised controlled clinical trial of adjuvant physical therapy versus occupational therapy. Pain. 1999;83:77-83.

18. Hakkaart-van Roijen I, van der Linden L, Bouwmans C, et al. [Kostenhandleiding. Methodologie van kostenonderzoek en referentieprijzen voor economische evaluaties in de gezondheidszorg]. Zorginstituut Nederland; 2015.

19. Goossens ME, Rutten-van Molken MP, Vlaeyen JW, et al. The cost diary: A method to measure direct and indirect costs in cost-effectiveness research. J Clin Epidemiol. 2000;53:688-695.

20. Drummond M, Sculpher M, Torrance G, et al. Methods for the economic evaluation of health care programmes. Oxford: Oxford University Press; 2005.

21. Moulaert VR, Goossens M, Heijnders IL, et al. Early neurologically focused follow-up after cardiac arrest is cost-effective: A trial-based economic evaluation. Resuscitation. 2016;106:30-36.
22. Koopmanschap MA, Rutten FF. Indirect costs in economic studies: Confronting the confusion. Pharmacoeconomics. 1993;4:446-454.

23. Aaronson NK, Muller M, Cohen PDA, et al. Translation, validation, and norming of the Dutch language version of the SF-36 Health Survey in Community and Chronic Disease Populations. $J$ Clin Epidemiol. 1998;51:1055-1068.

24. Ware JE, Kosinski M, Keller SK. SF-36 ${ }^{\circledR}$ Physical and Mental Health Summary Scales. 2 ed. Boston, MA: The Health Institute; 1994.

25. Brazier J, Roberts J, Deverill M. The estimation of a preference-based measure of health from the SF-36. J Health Econ. 2002;21:271-292.

26. Dolan P. Modeling valuations for EuroQol health states. Med Care. 1997;35:1095-1108.

27. Hendriks MR, Al MJ, Bleijlevens MH, et al. Continuous versus intermittent data collection of health care utilization. Med Decis Making. 2013;33:998-1008.

28. Drummond M, Manca A, Sculpher M. Increasing the generalizability of economic evaluations: Recommendations for the design, analysis, and reporting of studies. Int J Technol Assess Health Care. 2005;21:165-171.

29. Goossens MEJB, de Kinderen RJEM, Leeuw M, et al. Is exposure in vivo cost-effective for chronic low back pain? A trial-based economic evaluation. BMC Health Serv Res. 2015;15:549.

30. Leeuw M, Goossens MEJB, van Breukelen GJP, et al. Exposure in vivo versus operant graded activity in chronic low back pain patients: Results of a randomized controlled trial. Pain. 2008;138:192-207.

31. de Jong JR, Vlaeyen JWS, Onghena P, et al. Reduction of pain-related fear in complex regional pain syndrome type I: The application of graded exposure in vivo. Pain. 2005;116:264-275.

32. den Hollander M, de Jong JR, Volders S, et al. Fear reduction in patients with chronic pain: A learning theory perspective. Expert Rev Neurother. 2010;10:1733-1745. 